\title{
Limiting behavior of blow-up solutions for the cubic nonlinear beam equation
}

Pengshe Zheng ${ }^{1}$ and Lihui Leng ${ }^{1 *}$

"Correspondence:

lihuilengmath@163.com

${ }^{1}$ College of Science, Xihua

University, Chengdu, China

\section{Springer}

\begin{abstract}
In terms of the sharp Gagliardo-Nirenberg-Sobolev inequalities, we find the precisely sharp criteria of blow-up and global existence for the cubic nonlinear beam equations with $L^{2}$ critical nonlinearities. And we further study the limiting profile of blow-up solutions.
\end{abstract}

MSC: 35G20; 35Q40; 35B40

Keywords: Nonlinear beam equation; Blow-up solution; Sharp criteria; Limiting profile

\section{Introduction}

The nonlinear beam equation is a class of fourth-order partial differential equations appearing in different physical settings (see $[26,27]$ for a review), and it models the weak interactions of dispersive waves in [1] and the motion of the clamped plate and beams in [20]. In general, the nonlinear beam equation is in the following form:

$$
\frac{\partial^{2}}{\partial t^{2}} u+\Delta^{2} u+m u-|u|^{p-1} u=0, \quad x \in \mathbb{R}^{D},
$$

where the parameter $m>0 . u=u(t, x):[0, T) \times \mathbb{R}^{D} \rightarrow \mathbb{R}$ and $0<T \leq+\infty . \Delta^{2}=\Delta \Delta$ and $\Delta$ is the Laplacian. $1<p \leq \frac{2 D}{(D-4)^{+}}-1$, where $\frac{2 D}{(D-4)^{+}}=+\infty$ when $D=1,2,3,4 ; \frac{2 D}{(D-4)^{+}}=\frac{2 D}{D-4}$ when $D \geq 5$. When $m=0$, the scaling symmetry $\lambda^{\frac{4}{p-1}} u\left(\lambda^{2} t, \lambda x\right)$ of Eq. (1.1) implies two critical exponents: one is the $L^{2}$ critical exponent $p=1+\frac{8}{D}$, and the other is the $H^{2}$ energycritical exponent $p=\frac{2 D}{D-4}-1$ (see [12]). Hence, when $p=3$ and $D=4,6$, 8, we call Eq. (1.1) the $L^{2}$ critical, $L^{2}$ super-critical, and $H^{2}$ energy-critical cubic nonlinear beam equation, respectively. We supplement Eq. (1.1) with the initial data

$$
u(0, x)=u_{0}, \quad \frac{\partial}{\partial t} u(0, x)=u_{1} .
$$

In the last two decades, Eq. (1.1) has been widely studied. The local well-posedness of the Cauchy problem (1.1)-(1.2) for $1<p<\frac{2 D}{(D-4)^{+}}-1$ and $p=\frac{2 D}{(D-4)^{+}}-1$ was established in [17] and in [12], respectively. The stability of traveling waves and standing waves for Eq. (1.1) was obtained in [17]. There are a lot of papers on the asymptotic behavior and scattering properties of global solutions (see $[9,10,15,18,21,24,25,28,31,32])$. To our

(c) The Author(s) 2018. This article is distributed under the terms of the Creative Commons Attribution 4.0 International License (http://creativecommons.org/licenses/by/4.0/), which permits unrestricted use, distribution, and reproduction in any medium, provided you give appropriate credit to the original author(s) and the source, provide a link to the Creative Commons license, and indicate if changes were made. 
knowledge, the only result on the blow-up solutions of Eq. (1.1) is [12], in which authors gave a sufficient condition for the existence of blow-up solutions. This motivates us to study the following properties of Eq. (1.1): How to separate the domains of blow-up and global existence? What is the limiting behavior of blow-up solutions?

In terms of the sharp criteria for the nonlinear Schrödinger equation in $[8,14,16,19,23$, 36], we study the sharp criteria for the nonlinear Beam equation. First, for the $L^{2}$ critical case: $p=3$ and $D=4$, we have the following theorem.

Theorem 1.1 Let $m=1, p=3$, and $D=4$. Let $R$ be a ground state of

$$
\frac{3}{2} \Delta^{2} R+\frac{1}{2} R-|R|^{2} R=0, \quad R \in H^{2} .
$$

If the initial data $\left(u_{0}, u_{1}\right) \in H^{2} \times L^{2}$ satisfies

$$
E\left(\left(u_{0}, u_{1}\right)\right)<E(R, 0)=\frac{1}{2}\|R\|_{2}^{2},
$$

then we have the following:

(i) If $\left\|u_{0}\right\|_{2}<\|R\|_{2}$, then the solution $u(t, x)$ of the Cauchy problem (1.1)-(1.2) exists globally, and $u(t, x)$ satisfies that, for all time $t$,

$$
\|u(t)\|_{2}^{2}+\left\|\frac{\partial}{\partial t} u(t)\right\|_{2}^{2}<\|R\|_{2}^{2} \text { and }\|\Delta u(t)\|_{2}^{2}+\left\|\frac{\partial}{\partial t} u(t)\right\|_{2}^{2}<\|R\|_{2}^{2} .
$$

(ii) If $\left\|u_{0}\right\|_{2}>\|R\|_{2}$, then the solution $u(t, x)$ of the Cauchy problem (1.1)-(1.2) blows up in finite time $0<T<+\infty$.

The above sharp criteria of Eq. (1.1) are different from those of the $L^{2}$ critical nonlinear Schrödinger equation in [30], where Weinstein proved that for the initial data $\left\|u_{0}\right\|_{2}>$ $\|Q\|_{2}$, the corresponding solution may blow up. However, for Eq. (1.1), in the case $\left\|u_{0}\right\|_{2}>$ $\|R\|_{2}$, the corresponding solution must blow up in a finite time.

Furthermore, we obtain the following limiting profile of blow-up solutions for Eq. (1.1).

Theorem 1.2 Let $m=1, p=3$, and $D=4$. If $\left(u_{0}, u_{1}\right) \in H^{2} \times L^{2}$ and $u(t, x)$ is the corresponding blow-up solution of the Cauchy problem (1.1)-(1.2) with $\lim _{t \rightarrow T}\|u(t)\|_{2}=+\infty$ and $\|\Delta u(t)\|_{2}=\|\Delta R\|_{2}$, where $0<T<+\infty$ is the blow-up time, then there exist $y(t) \in \mathbb{R}^{4}$, $\gamma(t) \in \mathbb{R}$ such that

$$
u(t, \lambda(t)(\cdot+y(t))) \rightarrow R(\cdot) \quad \text { strongly in } H^{2} \text { as } t \rightarrow T,
$$

where $\lambda(t)=\left(\frac{\|u(t)\|_{2}}{\|R\|_{2}}\right)^{\frac{1}{2}}$ and $R$ is a ground state of $(1.3)$.

Throughout this paper, we assume $m=1$ and $p=3$ for simplification, and the general cases $m=1$ and $1<p<\frac{2 D}{(D-4)^{+}}-1$ can be obtained by entirely the same way. We extend the sharp criteria argument in $[14,16]$ for nonlinear Schrödinger equations to that for the nonlinear beam equation (1.1). This is very nontrivial because Eq. (1.1) does not have the conservation of $L^{2}$-norm and scaling invariance. The argument in this paper may have a potential application to other nonlinear wave equations without scaling invariance. 
In this paper, we abbreviate $L^{q}\left(\mathbb{R}^{D}\right),\|\cdot\|_{L^{q}\left(\mathbb{R}^{D}\right)}, H^{2}\left(\mathbb{R}^{D}\right)$, and $\int_{\mathbb{R}^{D}} \cdot d x$ by $L^{q},\|\cdot\|_{q}, H^{2}$, and $\int \cdot d x$. The various positive constants will be denoted by $C$.

\section{Notations and preliminaries}

For the Cauchy problem (1.1)-(1.2), the work space is defined by

$$
H^{2}:=\left\{v \in L^{2} \mid \int\left(|v|^{2}+|\nabla v|^{2}+|\Delta v|^{2}\right) d x<+\infty\right\}
$$

It is easy to check that $\left(\|v\|_{2}^{2}+\|\Delta v\|_{2}^{2}\right)^{\frac{1}{2}}$ is an equivalent norm of $H^{2}$, and this equivalent norm is used to decompose the bounded sequences in $H^{2}$ (see [37]). Define two functionals in $H^{2} \times L^{2}$ by

$$
\begin{aligned}
& E\left(\left(v(t), \frac{\partial}{\partial t} v(t)\right)\right):=\int\left[\frac{1}{2}\left|\frac{\partial}{\partial t} v(t)\right|^{2}+\frac{1}{2}|\Delta v(t)|^{2}+\frac{1}{2}|v(t)|^{2}-\frac{1}{4}|v(t)|^{4}\right] d x, \\
& H(v(t)):=\int\left[\frac{1}{2}|v(t)|^{2} d x-\frac{1}{4}|v(t)|^{4}\right] d x .
\end{aligned}
$$

The functionals $E$ and $H$ are well-defined by the Sobolev embedding theorem (see [12]). Moreover, Hebey and Pausader established the local well-posedness of the Cauchy problem (1.1)-(1.2) in the energy space $H^{2} \times L^{2}$ in [12] as follows.

Proposition 2.1 Let $m=1,1<p<\frac{2 D}{(D-4)^{+}}-1$, and $\left(u_{0}, u_{1}\right) \in H^{2} \times L^{2}$. There exists a unique solution $u(t, x)$ of the Cauchy problem (1.1)-(1.2) on the maximal time [0,T) such that $u(t, x) \in C\left([0, T) ; H^{2} \times L^{2}\right)$. Moreover, the following properties hold: either $T=+\infty($ global existence), or $0<T<+\infty$ and $\lim _{t \rightarrow T}\|u(t, x)\|_{H^{2}}=+\infty$ (blow-up). Furthermore, for all $t \in[0, T)$,

$$
E\left(\left(u(t), \frac{\partial}{\partial t} u(t)\right)\right)=E\left(\left(u_{0}, u_{1}\right)\right)
$$

Remark 2.2 Particularly, in the $H^{2}$ energy-critical case: $p=\frac{2 D}{(D-4)^{+}}-1$, Hebey and Pausader's result in [12] implies that the local well-posedness of the Cauchy problem (1.1)(1.2) also holds in $H^{2} \times L^{2}$. Moreover, for the solution $u(t, x) \in C\left([0, T) ; H^{2} \times L^{2}\right)$, if $0<T<+\infty$, then $\lim _{t \rightarrow T}\|u(t, x)\|_{H^{2}}=+\infty$, or $\lim \sup _{t \rightarrow T}\|u(t, x)\|_{L_{t}^{q}\left([0, T) ; L_{x}^{r}\right)}=+\infty$ (blowup), where

$$
(q, r)=\left(\frac{2(D+4)}{D-4}, \frac{2 D(D+4)}{(D-4)(D+2)}\right)
$$

is the $B$-admissible.

Now, we introduce the following important sharp inequalities: the sharp generalized Gagliardo-Nirenberg inequality established in [7] and in [37, 38]. Some other sharp Gagliardo-Nirenberg inequalities can be established by the profile arguments (see [2-4, $11,22,29,34,35])$. 
Lemma 2.3 Let $R$ be a ground state of (1.3) Then, when $D=4$, for all $v \in H^{2}$, we have

$$
\|v\|_{4}^{4} \leq \frac{2}{\|R\|_{2}^{2}}\|v\|_{2}^{2}\|\Delta v\|_{2}^{2}
$$

\section{Sharp criteria of blow-up and global existence}

In terms of the sharp Gagliardo-Nirenberg type inequalities, sharp Sobolev inequality, and some new estimates, we obtain the precisely sharp criteria of blow-up for Eq. (1.1): if $\left\|u_{0}\right\|_{2}<\|R\|_{2}$, then the solution exists globally; if $\left\|u_{0}\right\|_{2}>\|R\|_{2}$, then the solution blows up in finite time, where $R$ is a ground state of (1.3). Now, we give the proof of Theorem 1.1.

Proof (i) Applying the sharp inequality (2.2) to the energy functional $E$, we deduce that, for all $t \in I$ (maximal existence interval),

$$
\begin{aligned}
2 E\left(\left(u(t), \frac{\partial}{\partial t} u(t)\right)\right) & =\left\|\frac{\partial}{\partial t} u(t)\right\|_{2}^{2}+\|u(t)\|_{2}^{2}+\|\Delta u(t)\|_{2}^{2}-\frac{1}{2}\|u(t)\|_{4}^{4} \\
& \geq\left\|\frac{\partial}{\partial t} u(t)\right\|_{2}^{2}+\|u(t)\|_{2}^{2}+\left(1-\frac{\|u(t)\|_{2}^{2}}{\|R\|_{2}^{2}}\right)\|\Delta u(t)\|_{2}^{2} .
\end{aligned}
$$

By the bootstrap and continuity argument, we claim that if $\left\|u_{0}\right\|_{2}<\|R\|_{2}$, then, for all $t \in I$,

$$
\|u(t)\|_{2}<\|R\|_{2}
$$

Indeed, if (3.2) is not true, then there exists $t_{1} \in I$ such that $\left\|u\left(t_{1}\right)\right\|_{2} \geq\|R\|_{2}$. Since the solution $u(t, x)$ is continuous with respect to $t$, there exists $0<t_{0} \leq t_{1}$ such that $\left\|u\left(t_{0}\right)\right\|_{2}=$ $\|R\|_{2}$. But from (1.4), (3.1), and the conservation of energy $E\left(\left(u_{0}, u_{1}\right)\right)=E\left(\left(u(t), \frac{\partial}{\partial t} u(t)\right)\right)$ with $t=t_{0}$, we get

$$
\|R\|_{2}^{2}>2 E\left(\left(u\left(t_{0}\right), \frac{\partial}{\partial t} u\left(t_{0}\right)\right)\right) \geq\left\|\frac{\partial}{\partial t} u\left(t_{0}\right)\right\|_{2}^{2}+\left\|u\left(t_{0}\right)\right\|_{2}^{2} \geq\left\|u\left(t_{0}\right)\right\|_{2}^{2} .
$$

This contradicts $\left\|u\left(t_{0}\right)\right\|_{2}=\|R\|_{2}$. Hence, claim (3.2) holds.

Now, we can prove (1.5). By injecting (3.2) into (3.1), we can obtain the first estimate in (1.5) by (1.4) and (3.1). For the second estimate in (1.5), from (3.1), we see that $\forall t \in I$

$$
\|R\|_{2}^{2}>2 E\left(\left(u(t), \frac{\partial}{\partial t} u(t)\right)\right) \geq\|u(t)\|_{2}^{2}+\frac{\|R\|_{2}^{2}-\|u(t)\|_{2}^{2}}{\|R\|_{2}^{2}}\|\Delta u(t)\|_{2}^{2},
$$

and so, for all $t \in I$,

$$
\left(\|R\|_{2}^{2}-\|u(t)\|_{2}^{2}\right)\left(\|R\|_{2}^{2}-\|\Delta u(t)\|_{2}^{2}\right) \geq 0
$$

Inject (3.2) into (3.3). We get $\|\Delta u(t)\|_{2}^{2}<\|R\|_{2}^{2}$ for all $t \in I$. Moreover, by rewriting (3.1), we see that, for all $t \in I$,

$$
\begin{aligned}
\|R\|_{2}^{2} & >2 E\left(\left(u(t), \frac{\partial}{\partial t} u(t)\right)\right) \geq\left\|\frac{\partial}{\partial t} u(t)\right\|_{2}^{2}+\|\Delta u(t)\|_{2}^{2}+\left(1-\frac{\|\Delta u(t)\|_{2}^{2}}{\|R\|_{2}^{2}}\right)\|u(t)\|_{2}^{2} \\
& \geq\left\|\frac{\partial}{\partial t} u(t)\right\|_{2}^{2}+\|\Delta u(t)\|_{2}^{2} .
\end{aligned}
$$

Then the second estimate in (1.5) is true. 
(ii) We claim that if $\left\|u_{0}\right\|_{2}>\|R\|_{2}$, then for all $t \in I$

$$
\|u(t)\|_{2}>\|R\|_{2} \quad \text { and } \quad\|\Delta u(t)\|_{2}>\|R\|_{2} .
$$

Indeed, if $\|u(t)\|_{2}>\|R\|_{2}$ is not true for all $t \in I$, then there exists $t_{2} \in I$ such that $\left\|u\left(t_{2}\right)\right\|_{2} \leq$ $\|R\|_{2}$. From the continuity of $\|u(t)\|_{2}$, there exists $0<t_{3} \leq t_{2}$ such that $\left\|u\left(t_{3}\right)\right\|_{2}=\|R\|_{2}$. Injecting this into (3.1) with $t=t_{3}$, we get

$$
2 E\left(\left(u\left(t_{3}\right), \frac{\partial}{\partial t} u\left(t_{3}\right)\right)\right) \geq\left\|u\left(t_{3}\right)\right\|_{2}^{2}=\|R\|_{2}^{2},
$$

which contradicts $E\left(\left(u(t), \frac{\partial}{\partial t} u(t)\right)\right)=E\left(\left(u_{0}, u_{1}\right)\right)<\frac{1}{2}\|R\|_{2}^{2}$ for all $t \in I$. Then we prove that $\|u(t)\|_{2}>\|R\|_{2}$ for all $t \in I$. Moreover, injecting $\|u(t)\|_{2}>\|R\|_{2}$ for all $t \in I$ into (3.3), we obtain that $\|\Delta u(t)\|_{2}>\|R\|_{2}$ for all $t \in I$. This completes the proof of claim (3.4). Now, let $J(t):=\int|u(t, x)|^{2} d x$. By some basic computations, we deduce that, for all $t \in I, J^{\prime}(t)=$ $2 \int u(t) \frac{\partial}{\partial t} u(t) d x$ and

$$
\begin{aligned}
J^{\prime \prime}(t) & =6\left\|\frac{\partial}{\partial t} u(t)\right\|_{2}^{2}+2\|u(t)\|_{2}^{2}+2\|\Delta u(t)\|_{2}^{2}-8 E\left(\left(u_{0}, u_{1}\right)\right) \\
& >6\left\|\frac{\partial}{\partial t} u(t)\right\|_{2}^{2}
\end{aligned}
$$

where the last step employs (1.4) and (3.4). Hence, $J^{\prime \prime}(t)$ is positive and has a lower bound for all $t \in I$. Notice that $J^{\prime}(t)^{2} \leq 4\|u(t)\|_{2}^{2}\left\|\frac{\partial}{\partial t} u(t)\right\|_{2}^{2}$. Then

$$
J(t) J^{\prime \prime}(t)>6\left\|\frac{\partial}{\partial t} u(t)\right\|_{2}^{2}\|u(t)\|_{2}^{2}>\frac{3}{2} J^{\prime}(t)^{2} .
$$

There exists $t_{0}>0$ such that $J^{\prime}(t)>0$ for all $t>t_{0}$. Thus, for all $t>t_{0}$, we get $\frac{J^{\prime \prime}(t)}{J^{\prime}(t)}>\frac{3}{2} \frac{J^{\prime}(t)}{J(t)}$, which implies that there exists $K>0$ such that $J^{\prime}(t)>K J(t)^{\frac{3}{2}}$. Due to $\frac{3}{2}>1$, we deduce that, for all $t>t_{0}$,

$$
J(t)>\left(\frac{2 \sqrt{J\left(t_{0}\right)}}{2-K \sqrt{J\left(t_{0}\right)}\left(t-t_{0}\right)}\right)^{2} .
$$

Then there exists $0<T<+\infty$ such that $\lim _{t \rightarrow T}\|u(t)\|_{2}^{2}=\lim _{t \rightarrow T} J(t)=+\infty$. The solution $u(t, x)$ blows up in finite time $0<T<+\infty$.

\section{Limiting profile of blow-up solutions}

In this section, we assume that the ground state of (1.3) is unique up to translations in space and dilations, which is also denoted by $R$. A similar assumption has been used for the ground state of the classical second-order nonlinear Schrödinger equation in [30]. First, we show the variational characteristics of $R$.

Lemma 4.1 Let $D=4$. If $v \in H^{2}$ satisfies $\|\Delta v\|_{2}=\|\Delta R\|_{2}$ and $H(v)=0$, then $v(x)$ is of the following form:

$$
v(x)=R\left(\lambda x+x_{0}\right) \text { for some } \lambda>0, x_{0} \in \mathbb{R}^{4},
$$

where $R$ is a ground state of (1.3). 
Proof According to the hypothesis $H(v)=0$, we see that $\int|v|^{2} d x=\frac{1}{2} \int|v|^{4} d x$. Hence, inject this into the following functional:

$$
I(v):=\frac{\left(\int|v|^{2} d x\right)\left(\int|\Delta v|^{2} d x\right)}{\int|v|^{4} d x}=\frac{1}{2}\|\Delta v\|_{2}^{2}=\frac{1}{2}\|\Delta R\|_{2}^{2}=\frac{1}{2}\|R\|_{2}^{2}=I_{*},
$$

which implies that $v$ is a minimizer of $I(v)$. According to Zhu, Zhang, and Yang's result in [37], we can deduce that $v(x)$ is of the form $v(x)=R\left(\lambda x+x_{0}\right)$ by the uniqueness of $R$.

The main tool to study the limit of the blow-up solutions for Eq. (1.1) is the profile decomposition established by Zhu, Zhang, and Yang in [37]. This argument has been applied to study the stability of standing waves (see $[5,6,33])$.

Proposition 4.2 Let $D=4$ and $\left\{v_{n}\right\}_{n=1}^{+\infty}$ be a bounded sequence in $H^{2}$. Then there exist a subsequence of $\left\{v_{n}\right\}_{n=1}^{+\infty}\left(\right.$ still denoted $\left.\left\{v_{n}\right\}_{n=1}^{+\infty}\right)$, a family $\left\{x_{n}^{j}\right\}_{j=1}^{+\infty}$ of sequences in $\mathbb{R}^{4}$, and a sequence $\left\{V^{j}\right\}_{j=1}^{+\infty}$ in $H^{2}$ such that

(i) for every $k \neq j,\left|x_{n}^{k}-x_{n}^{j}\right| \rightarrow+\infty$ as $n \rightarrow+\infty$;

(ii) for every $l \geq 1$ and every $x \in \mathbb{R}^{4}, v_{n}(x)=\sum_{j=1}^{l} V^{j}\left(x-x_{n}^{j}\right)+v_{n}^{l}(x)$ with $\lim _{l \rightarrow+\infty} \lim \sup _{n \rightarrow+\infty}\left\|v_{n}^{l}\right\|_{q}=0$ for every $q \in(2,+\infty)$.

Moreover, as $n \rightarrow+\infty$, we have

$$
\begin{aligned}
& \left\|v_{n}\right\|_{2}^{2}=\sum_{j=1}^{l}\left\|V^{j}\right\|_{2}^{2}+\left\|v_{n}^{l}\right\|_{2}^{2}+o(1), \\
& \left\|\Delta v_{n}\right\|_{2}^{2}=\sum_{j=1}^{l}\left\|\Delta V^{j}\right\|_{2}^{2}+\left\|\Delta v_{n}^{l}\right\|_{2}^{2}+o(1), \\
& \left\|\sum_{j=1}^{l} V^{j}\left(x-x_{n}^{j}\right)\right\|_{4}^{4}=\sum_{j=1}^{l}\left\|V^{j}\left(x-x_{n}^{j}\right)\right\|_{4}^{4}+o(1),
\end{aligned}
$$

where $o(1):=o_{n}(1) \rightarrow 0$ as $n \rightarrow+\infty$.

Remark 4.3 By using the inequality

$$
\left.|| \sum_{j=1}^{l} a_{j}\right|^{p+1}-\left.\sum_{j=1}^{l}\left|a_{j}\right|^{p+1}\left|\leq C \sum_{j \neq k}\right| a_{j}|| a_{k}\right|^{p}
$$

for $p>1$, we can prove that the mixed terms in $\left\|\sum_{j=1}^{l} V^{j}\left(x-x_{n}^{j}\right)\right\|_{p+1}^{p+1}$ vanish. Thus, (4.2) is true.

In terms of Hmidi and Keraani's argument in [13] and Zhu, Zhang, and Yang's argument in [37], we prove the following refined compactness result by Proposition 4.2.

Lemma 4.4 Let $D=4$ and $\left\{v_{n}\right\}_{n=1}^{+\infty}$ be a bounded sequence in $H^{2}$ such that

$$
\limsup _{n \rightarrow+\infty}\left\|v_{n}\right\|_{2} \leq M \quad \text { and } \quad \limsup _{n \rightarrow+\infty}\left\|v_{n}\right\|_{4} \geq N>0 .
$$


Then there exists a sequence $\left\{x_{n}\right\}_{n=1}^{+\infty}$ of $\mathbb{R}^{4}$ such that up to a subsequence

$$
v_{n}\left(x+x_{n}\right) \rightarrow V(x) \quad \text { weakly in } H^{2}
$$

with $\|\Delta V\|_{2}^{2} \geq \frac{\|\Delta R\|_{2}^{2} N^{4}}{2 M^{2}}$, and $R$ is a ground state of (1.3).

Proof The proof of Lemma 4.4 is fully similar to that of Theorem 1.1 in [37]. The key is injecting the decomposition: $v_{n}(x)=\sum_{j=1}^{l} V^{j}\left(x-x_{n}^{j}\right)+v_{n}^{l}(x)$ into (4.3). Indeed, from (4.2), we deduce that as $n \rightarrow+\infty, l \rightarrow+\infty$,

$$
N^{4} \leq \limsup _{n \rightarrow+\infty}\left(\left\|\sum_{j=1}^{l} V^{j}\left(x-x_{n}^{j}\right)\right\|_{4}+\left\|v_{n}^{l}(x)\right\|_{4}\right)^{4} \leq \sum_{j=1}^{+\infty}\left\|V^{j}\right\|_{4}^{4} .
$$

The left proof is similar to the proof of Theorem 1.1 in [37], and is omitted.

Applying Lemma 4.4, we obtain the following limiting profile of blow-up solutions for Eq. (1.1) in Theorem 1.2: for the blow-up solution of the Cauchy problem (1.1)-(1.2) satisfying $\lim _{t \rightarrow T}\|u(t)\|_{2}=+\infty$ and $\|\Delta u(t)\|_{2}=\|\Delta R\|_{2}$, where $0<T<+\infty$ is the blow-up time, we prove that $u(t, x)$ remains close to the ground state $R$ in $H^{2}$ up to scaling and translation in the nonradial case, where $R$ is the ground state of (1.3).

Proof of Theorem 1.2 By the assumptions, for any $t_{n} \rightarrow T$ as $n \rightarrow+\infty$, take

$$
\lambda_{n}^{2}:=\frac{\left\|u\left(t_{n}, x\right)\right\|_{2}}{\|R\|_{2}} \rightarrow+\infty \quad \text { as } n \rightarrow+\infty,
$$

and $U_{n}=u\left(t_{n}, \lambda_{n} x\right)$. We note that

$$
\left\{\begin{array}{l}
\left\|U_{n}\right\|_{2}=\frac{1}{\lambda_{n}^{2}}\left\|u\left(t_{n}\right)\right\|_{2}=\|R\|_{2}, \\
\left\|\Delta U_{n}\right\|_{2}=\left\|\Delta u\left(t_{n}\right)\right\|_{2}=\|\Delta R\|_{2} .
\end{array}\right.
$$

Therefore, $\left\{U_{n}\right\}_{n=1}^{+\infty}$ is a uniformly bounded sequence in $H^{2}$ and $\left\{U_{n}\right\}_{n=1}^{+\infty}$ has a weakly convergent subsequence $\left\{U_{n}\right\}_{n=1}^{+\infty}$ (still denoted by $\left.\left\{U_{n}\right\}_{n=1}^{+\infty}\right)$. And for the subsequence $\left\{U_{n}\right\}_{n=1}^{+\infty}$, we deduce that

$$
\begin{aligned}
H\left(U_{n}\right) & =\frac{1}{\lambda_{n}^{4}}\left(E\left(\left(u_{0}, u_{1}\right)\right)-\frac{1}{2}\left\|\frac{\partial}{\partial t} u\left(t_{n}\right)\right\|_{2}^{2}-\frac{1}{2}\left\|\Delta u\left(t_{n}\right)\right\|_{2}^{2}\right) \\
& \leq \frac{1}{\lambda_{n}^{4}} E\left(\left(u_{0}, u_{1}\right)\right) \rightarrow 0 \text { as } n \rightarrow+\infty .
\end{aligned}
$$

Meanwhile, from (2.2), we see that $\left\|\Delta U_{n}\right\|_{2}=\|\Delta R\|_{2}$ implies

$$
H\left(U_{n}\right) \geq \frac{1}{2}\left(1-\frac{\left\|\Delta U_{n}\right\|_{2}^{2}}{\|\Delta R\|_{2}^{2}}\right)\left\|U_{n}\right\|_{2}^{2} \geq 0
$$

Then we get $\lim _{n \rightarrow+\infty} H\left(U_{n}\right)=0$ and $\lim _{n \rightarrow+\infty}\left\|U_{n}\right\|_{4}^{4}=2\|R\|_{2}^{2}$. By applying Lemma 4.4 to the sequence $\left\{U_{n}\right\}_{n=1}^{+\infty}$ (here, we take $M^{2}=\|R\|_{2}^{2}, N^{4}=2\|R\|_{2}^{2}$ ), there exist $\left\{y_{n}\right\}_{n=1}^{+\infty} \subset \mathbb{R}^{4}$ and 
$U(x) \in H^{2}$ such that

$$
U_{n}\left(x+y_{n}\right) \rightarrow U(x) \quad \text { weakly in } H^{2} \text { as } n \rightarrow+\infty
$$

with $\|\Delta U\|_{2} \geq\|\Delta R\|_{2}$. But by the lower semi-continuity of norm, we get $\|\Delta U\|_{2} \leq$ $\liminf _{n \rightarrow+\infty}\left\|\Delta U_{n}\left(x+y_{n}\right)\right\|_{2}=\|\Delta R\|_{2}$, and so we get $\|\Delta U\|_{2}=\|\Delta R\|_{2}=\left\|\Delta U_{n}\left(x+y_{n}\right)\right\|_{2}$. And from the Brézis-Lieb lemma, we get

$$
\lim _{n \rightarrow+\infty}\left\|\Delta\left(U_{n}\left(x+y_{n}\right)-U(x)\right)\right\|_{2}=0
$$

Applying (2.2) to $U_{n}\left(x+y_{n}\right)-U$, there exists $C>0$ such that

$$
\left\|U_{n}\left(x+y_{n}\right)-U\right\|_{4}^{4} \leq C\left\|U_{n}\left(x+y_{n}\right)-U\right\|_{2}^{2}\left\|\Delta\left(U_{n}\left(x+y_{n}\right)-U\right)\right\|_{2}^{2} .
$$

Inject (4.6) and $\left\|U_{n}\left(x+y_{n}\right)-U(x)\right\|_{2} \leq 2\|R\|_{2}$ into the above estimate.

$$
U_{n}\left(x+y_{n}\right) \rightarrow U(x) \quad \text { strongly in } L^{4} \text { as } n \rightarrow+\infty
$$

Then we have proved that $\lim _{n \rightarrow+\infty} H\left(U_{n}\left(x+y_{n}\right)\right)=H(U)=0$, which implies that $\|U\|_{2}^{2}=$ $\|R\|_{2}^{2}$, and so, from the Brézis-Lieb lemma, (4.6), and (4.9), we see that

$$
U_{n}\left(x+y_{n}\right) \rightarrow U(x) \quad \text { strongly in } H^{2} \text { as } n \rightarrow+\infty .
$$

Now, collecting the properties of $U=U(x)$, we see that

$$
U \in H^{2}, \quad\|U\|_{2}=\|R\|_{2}, \quad\|\Delta U\|_{2}=\|\Delta R\|_{2} \quad \text { and } \quad H(U)=0 .
$$

Applying the variational characteristic of the ground state (see Lemma 4.1), there exist $\lambda_{0}>0$ and $x_{0} \in \mathbb{R}^{4}$ such that $U(x)=U\left(\lambda_{0} x+x_{0}\right)$, and so we can obtain (1.6) by redefining $\lambda(t)$ and $x(t)$.

\section{Acknowledgements}

We thank the referees for pointing out some misprints and helpful suggestions.

Funding

This work is supported by Sichuan Science and Technology Program of China (No. 2015JY0245), Scientific Research Fund of Sichuan Provincial Education Department of China (No. 15ZA0135, No. 18ZB0570), and the National Natural Science Foundation of China (No. 11371267).

\section{Abbreviations}

Not applicable.

\section{Availability of data and materials}

Data sharing not applicable to this article as no datasets were generated or analyzed during the current study.

\section{Competing interests}

The authors declare that they have no competing interests.

\section{Authors' contributions}

$\mathrm{PZ}$ and LL have the same contribution to this work. All authors read and approved the final manuscript.

\section{Publisher's Note}

Springer Nature remains neutral with regard to jurisdictional claims in published maps and institutional affiliations. 
References

1. Bretherton, F.P.: Resonant interaction between waves: the case of discrete oscillations. J. Fluid Mech. 20, 457-479 (1964)

2. Edmunds, D.E., Fortunato, D., Janelli, E.: Critical exponents, critical dimensions and the biharmonic operator. Arch Ration. Mech. Anal. 112, 269-289 (1990)

3. Feng, B.H.: On the blow-up solutions for the fractional nonlinear Schrödinger equation with combined power-type nonlinearities. Commun. Pure Appl. Anal. 17(5), 1785-1804 (2018)

4. Feng, B.H.: On the blow-up solutions for the nonlinear Schrödinger equation with combined power-type nonlinearities. J. Evol. Equ. 18, 203-220 (2018)

5. Feng, B.H., Zhang, H.H.: Stability of standing waves for the fractional Schrödinger-Choquard equation. Comput. Math. Appl. 75, 2499-2507 (2018)

6. Feng, B.H., Zhang, H.H.: Stability of standing waves for the fractional Schrödinger-Hartree equation. J. Math. Anal. Appl. 460, 352-364 (2018)

7. Fibich, G., Ilan, B., Papanicolaou, G.: Self-focusing with fourth-order dispersion. SIAM J. Appl. Math. 62, 1437-1462 (2002)

8. Goubet, O., Hamraoui, E.: Blow-up of solutions to cubic nonlinear Schrödinger equations with defect: the radial case. Adv. Nonlinear Anal. 6(2), 183-197 (2017)

9. Guo, A., Cui, S.: Global solutions and self-similar solutions for nonlinear beam equations. Math. Comput. Model. 44, 405-412 (2006)

10. Guo, A., Cui, S.: Solvability of the Cauchy problem of nonlinear beam equation in Besov spaces. Nonlinear Anal. 65 , 802-824 (2006)

11. Guo, Q., Zhu, S.H.: Sharp threshold of blow-up and scattering for the fractional Hartree equation. J. Differ. Equ. 264(4), 2802-2832 (2018)

12. Hebey, E., Pausader, B.: An introduction to fourth order nonlinear wave equations. http://www.math.brown.edu/ benoit/

13. Hmidi, T., Keraani, S.: Blowup theory for the critical nonlinear Schrödinger equations revisited. Int. Math. Res. Not. 46, 2815-2828 (2005)

14. Holmer, J., Roudenko, S.: On blow-up solutions to the 3D cubic nonlinear Schrödinger equation. Appl. Math. Res. Express 2007, Article ID 004 (2007)

15. Just, A., Stempień, Z:: Pareto optimal control problem and its Galerkin approximation for a nonlinear one-dimensional extensible beam equation. Opusc. Math. 36(2), 239-252 (2016)

16. Kenig, C., Merle, F.: Global well-posedness, scattering and blow-up for the energy-critical, focusing, non-linear Schrödinger equation in the radial case. Invent. Math. 166, 645-675 (2006)

17. Levandosky, S.P.: Stability and instability of fourth-order solitary waves. J. Dyn. Differ. Equ. 10, 151-188 (1998)

18. Levandosky, S.P., Strauss, W.A.: Time decay for the nonlinear beam equation. Methods Appl. Anal. 7, 479-488 (2000)

19. Li, X.G., Zhang, J.: Limit behavior of blow-up solutions for critical nonlinear Schrödinger equation with harmonic potential. Differ. Integral Equ. 19(7), 761-771 (2006)

20. Love, A.E.H.: A Treatise on the Mathematical Theory of Elasticity. Dover, New York (1944)

21. Miao, C.X.: A note on time decay for the nonlinear beam equation. J. Math. Anal. Appl. 314, 764-773 (2006)

22. Miao, C.X., Xu, G.X., Zhao, L.F.: Global well-posedness and scattering for the focusing energy-critical nonlinear Schrödinger equations of fourth order in the radial case. J. Differ. Equ. 246, 3715-3749 (2009)

23. Nawa, H.: Limiting profiles of blow-up solutions of the nonlinear Schrödinger equation with critical power nonlinearity. Proc. Jpn. Acad., Ser. A, Math. Sci. 73(10), 171-175 (1997)

24. Pausader, B.: Scattering and the Levandosky-Strauss conjecture for fourth order nonlinear wave equations. J. Differ. Equ. 241, 237-278 (2007)

25. Pausader, B.: Scattering for the beam equation in low dimension. Indiana Univ. Math. J. 59(3), 791-822 (2009)

26. Peletier, L., Troy, W.C.: Spatial Patterns Higher Order Models in Physics and Mechanics. Progress in Nonlinear Differential Equations and Their Applications, vol. 45. Birkhäuser, Basel (2001)

27. Radulescu, $\mathrm{V}$.: Singular phenomena in nonlinear elliptic problems: from blow-up boundary solutions to equations with singular nonlinearities. In: Handbook of Differential Equations: Stationary Partial Differential Equations. Handb. Differ. Equ., vol. IV, pp. 485-593. Elsevier, Amsterdam (2007)

28. Sun, Y.: Indirect boundary integral equation method for the Cauchy problem of the Laplace equation. J. Sci. Comput. 71, 469-498 (2017)

29. Talenti, G.: Best constant in Sobolev inequality. Ann. Mat. Pura Appl. 110, 353-372 (1976)

30. Weinstein, M.I.: Nonlinear Schrödinger equations and sharp interpolation estimates. Commun. Math. Phys. 87 567-576 (1983)

31. Xiang, M., Radulescu, V.D., Zhang, B.: Nonlocal Kirchhoff diffusion problems: local existence and blow-up of solutions. Nonlinearity 31, 3228-3250 (2018)

32. Xiang, M., Zhang, B., Qiu, H.: Existence of solutions for a critical fractional Kirchhoff type problem in $\mathbb{R}^{N}$. Sci. China Math. 60, 1647-1660 (2017)

33. Zhang, J., Zhu, S.H.: Stability of standing waves for the nonlinear fractional Schrödinger equation. J. Dyn. Differ. Equ. 29(3), 1017-1030 (2017)

34. Zhu, S.H.: On the blow-up solutions for the nonlinear fractional Schrödinger equation. J. Differ. Equ. 261(2), 1506-1531 (2016)

35. Zhu, S.H.: Existence of stable standing waves for the fractional Schrödinger equations with combined nonlinearities. J. Evol. Equ. 17(3), 1003-1021 (2017)

36. Zhu, S.H., Zhang, J.: Limiting behavior of blow-up solutions of the NLSE with a Stark potential. Acta Math. Sci. 32(3), $1181-1192(2012)$

37. Zhu, S.H., Zhang, J., Yang, H.: Limiting profile of the blow-up solutions for the fourth-order nonlinear Schrödinger equation. Dyn. Partial Differ. Equ. 7, 187-205 (2010)

38. Zhu, S.H., Zhang, J., Yang, H.: Biharmonic nonlinear Schrödinger equation and the profile decomposition. Nonlinear Anal. 74(17), 6244-6255 (2011) 